\title{
Intervenções farmacêuticas e adesão ao tratamento farmacológico em usuários do centro de atenção psicossocial para álcool e outras drogas
}

\author{
Pharmaceutical intervention and medication adherence in a \\ psychosocial care center for alcohol and other drugs
}

Jussara Secundo dos Santos'; Maraiza Alves de Oliveira²; Giselle de Carvalho Brito²; Chiara Ermínia da Rocha; Giuliano Di Pietro'

'Universidade Federal de Sergipe, São Cristóvão.

${ }^{2}$ Universidade Federal de Sergipe, Lagarto

\begin{abstract}
Resumo
Introdução: A não adesão ao tratamento farmacológico e não-farmacológico oferecidos nos centros de atenção psicossocial para álcool e outras drogas pode afetar diretamente a reabilitação dos usuários. Portanto, sugere-se que Intervenções farmacêuticas (IF) educativas podem promover o aumento da adesão ao tratamento, assim como a relação colaborativa do usuário na redução de danos. Objetivo: Avaliar o efeito das intervenções farmacêuticas educativas na adesão ao tratamento dos usuários do CAPSad. Metodologia: Utilizou-se a Escala de Adesão Terapêutica de quatro itens de Morisky (MMAS-4), modificada para este estudo antes e após as Intervenções Farmacêuticas (IF) para avaliar a adesão. Resultados: Observou-se o aumento da adesão após as IF de 10\% para 23,3\% dos usuários que apresentavam alta adesão, de 13,3\% para 26,7\% os de média adesão, com redução de $76,7 \%$ para $50 \%$ daqueles que apresentavam baixa adesão. Conclusão: Com apenas uma Intervenção Farmacêutica, o número de usuários do CAPSad que passaram a apresentar uma maior adesão ao tratamento medicamentoso teve um aumento significativo. Há uma grande carência de profissionais Farmacêuticos que atuam na área psicossocial, a inclusão deste profissional no Programa de Saúde da Família é necessária.
\end{abstract}

Palavras chave: psicotrópicos; álcool; drogas ilícitas; educação; saúde mental. 


\begin{abstract}
Introduction: Non-adherence to pharmacological and non-pharmacological treatment offered in psychosocial care centers for alcohol and other drugs (CAPSad) can directly affect the rehabilitation of users. Therefore, it is suggested that educational Pharmaceutical Interventions (PI) can promote increased adherence to treatment, as well as the user's collaborative relationship in harm reduction. Objective: To evaluate the effect of educational PI on adherence to treatment by CAPSad users. Methodology: We used the Morisky Therapeutic Adherence Scale of four items (MMAS-4), modified for this study before and after PI to assess adherence. Results: There was an increase in adherence after PI from $10 \%$ to $23.3 \%$ of users who had high adherence, from $13.3 \%$ to $26.7 \%$ those with medium adherence, with a reduction of $76.7 \%$ for $50 \%$ of those with low adherence. Conclusion: With only PI for 3 months, the number of CAPSad users who started to show greater adherence to drug treatment had a significant increase. There is a great lack of Pharmaceutical professionals who work in the psychosocial area, requiring the inclusion of this professional in the Family Health Program.
\end{abstract}

Keywords: psychotropic drugs; alcohol; illicit drugs; education; mental health.

Recebido em: 08-10-2020 Publicado em:29-10-2020

\title{
Autor correspondente
}

Giuliano Di Pietro

Av. Marechal Rondon s/n. Bairro Roza Elze,

São Cristóvão, Sergipe.

Email: dipietrobr@yahoo.com.br

\section{Introdução}

O consumo de substâncias psicoativas tem aumento nas últimas décadas e tem sido um desafio para a saúde pública em todo o mundo'. As doenças causadas pela dependência comprometem o dependente químico, sua saúde, a família e a dinâmica social. A prevenção é fundamental, mas ações que possam minimizar os impactos do uso de drogas em indivíduos, famílias e comunidades devem ser valorizadas².

Segundo a OMS, o consumo de álcool resulta em 3,3 milhões de mortes em todo o mundo'. Acredita-se que o consumo intenso de álcool seja um fator de risco para câncer ${ }^{3}$, além do risco de outras doenças não transmissíveis ${ }^{4}$, como transtornos mentais e comportamentais, e é também responsável por lesões, acidentes de trânsito e mortes ${ }^{5}$.

Com relação à cocaína, estima-se que 18,8 milhões de usuários consomem anualmente esta droga ${ }^{6}$. O distúrbio causado na vida do usuário crônico é caracterizado por comportamentos de busca, falta de controle, mau funcionamento social e uso arriscado da droga7. Quando a cocaína é fumada, consumido como crack cocaína, a retirada, os efeitos, e o prognóstico são muito piores do que quando a cocaína é inalada, por exemplo8. 
Nos EUA, o abuso de drogas tem sido uma grande preocupação pública há mais de meio século. Em 2016, aproximadamente 7,4 milhões de americanos com idade $\geq 12$ anos usaram drogas ilícitas ${ }^{9}$, além da recente epidemia de opioides que foi declarada como uma emergência nacional de saúde pública ${ }^{10}$.

$\bigcirc 3^{\circ}$ Levantamento sobre o uso de drogas pela população brasileira realizado pela Fiocruz, revela que 3,2\% da população usou substâncias ilícitas nos 12 meses anteriores à pesquisa, correspondendo a 4,9 milhões de brasileiros (homens: 5\% e mulheres 1,5\%), com maior incidência entre os jovens de 18 e 24 anos (7,4\%). A substância ilícita mais consumida no Brasil é a maconha, e 7,7\% da população entre 12 a 65 anos já a usaram ao menos uma vez na vida. Em segundo lugar (3,1\%), a cocaína em pó, e 0,9\% relataram ter feito uso de crack (homens 1,4\% e mulheres 0,4\%; 1,4 milhão de pessoas) $)^{17}$. percentual encontrado no $3^{\circ}$ Levantamento é inferior ao que aparece na Pesquisa Nacional do Uso do Crack ${ }^{12}$, justamente por ser domiciliar, enquanto os usuários de crack compõem uma população majoritariamente marginalizada, e que, muitas vezes, vive em situação de rua.

C Centro de Atenção Psicossocial ao Álcool e Outras Drogas (CAPSad) é um serviço de saúde para tratamento de transtornos causados pelo uso de álcool e problemas relacionados a drogas no Brasil e é oferecido pelo Sistema Público de Saúde (SUS), com atendimento diário (24h) aos usuários. O CAPSad oferece salas de repouso, condições de desintoxicação e é apoiado por uma unidade psiquiátrica, em um hospital geral, para pacientes que precisam ser internados ${ }^{13}$.

Medicamentos psicotrópicos são usados para reduzir os sintomas da droga e de abstinência, proporcionando desintoxicação e diminuição ou secessão do vício ${ }^{14}$. Os antidepressivos, por exemplo, são largamente prescritos a pacientes recebendo tratamento para dependência química ${ }^{15}$. Alguns estudos mostram que pacientes com transtorno grave do uso de álcool ou outras substâncias (SUD), apresentam também transtornos de humor ou transtornos de ansiedade ${ }^{16}$.

Entretanto pouco se tem estabelecido como tratamento medicamentoso padrão a pacientes com SUD. Estes tendem a ser distúrbios recidivantes crônicos em diferentes estágios, por exemplo, abstinência, recaída, crawing, prevenção, e podem precisar de tratamento diferenciado, com abordagens medicamentosas personalizadas. Além disso, ocorre plasticidade neuronal, após a exposição crônica a substâncias psicoativas ${ }^{17}$, e mudanças duradouras também aparecem, como alterações na função neurocognitiva, no humor, e ansiedade ${ }^{18}$.

A falta da correta adesão ao tratamento medicamentoso do paciente, geralmente, resulta na exacerbação de sintomas indesejados e efeitos adversos com intensidade significativa, levando ao abandono do tratamento pelo paciente, comprometendo a eficácia do tratamento e a recuperação do usuário de drogas $^{19}$. A pesquisa realizada por Schmidt e col., mostrou que menos de 10\% dos indivíduos com transtornos relacionados ao uso de álcool que desejam parar de beber no Brasil procuram tratamento ${ }^{20}$. As baixas taxas de utilização dos serviços especializados e adesão aos tratamentos para SUD ${ }^{21,22}$ são preocupantes. Os usuários de crack, em relação à cocaína inalada, por exemplo, apresentam maus resultados no tratamento ${ }^{23}$, com altas taxas de 
abandono ${ }^{24}$, e baixa abstinência póstratamento ${ }^{25}$.

Muitos são os obstáculos para a completa adesão ao tratamento SUD, aqueles relacionados ao próprio paciente como crenças pessoais, estigmas, preconceito, aspectos psicossociais ou do próprio equipamento de saúde, seus profissionais e procedimentos adotados ${ }^{26,27}$. O uso crônico de álcool e outras drogas geralmente está associado a outras comorbidades, necessitando de diferentes estratégias de tratamento como redução de danos, atendimento ambulatório e hospitalar, desintoxicação, acolhimento diurno e noturno, entretanto esses equipamentos, muitas vezes, não se conectam, dificultando a continuidade do tratamento e a maior adesão pelo paciente SUD ${ }^{28}$.

Com base nesses aspectos, o objetivo deste estudo foi avaliar a adesão de pacientes SUD de um CAPSad, antes e após intervenções farmacêuticas (IF), a fim de desenvolver estratégias para uma maior adesão dos pacientes SUD ao serviço e aos tratamentos medicamentosos e complementares, com o alvo na redução de danos.

\section{Metodologia}

\section{Desenho e configuração do estudo}

Foi realizado um estudo não controlado antes e depois da adesão aos medicamentos com pacientes do CAPSad, aplicando o instrumento MMAS$4^{29}$, traduzido e padronizado para 0 português por Remondi e colaboradores $^{30}$. $\bigcirc$ instrumento foi aplicado por acadêmicos da Faculdade de Farmácia, previamente treinados e acompanhados pelos preceptores da instituição. A Intervenção Farmacêutica
(IF) foi realizada durante 3 meses e o instrumento foi aplicado antes e depois disso. A pesquisa foi aprovada pelo Comitê de Ética em Pesquisa do Hospital Universitário da Universidade Federal de Sergipe, com o número CAAE: 92400618.4.0000.5546. Todos os participantes assinaram o termo de consentimento informando que foram coletados e armazenados, de acordo com os regulamentos de privacidade vigentes.

\section{População estudada}

Todos os pacientes registrados no CAPSad foram convidados a participar, porém apenas 30 completaram todas as etapas do estudo. $\bigcirc$ uso de medicação psicotrópica foi o principal critério de inclusão dos pacientes neste estudo. A verificação dessas informações foi realizada pela leitura dos prontuários dos pacientes, que foram examinados no momento após a aplicação dos instrumentos. Outros critérios de inclusão foram a idade de dezoito anos ou mais e a participação atual nas atividades do CAPSad. Foram excluídos os pacientes que não conseguiam se comunicar com clareza ou por estarem sob influência de álcool e drogas ilícitas no momento do estudo.

\section{Coleta de dados e medidas de resultados}

Primeiro, um instrumento foi utilizado para coletar informações sociodemográficas com variáveis independentes, como sexo, idade, escolaridade, ocupação atual, comportamentos relacionados à saúde e fatores de estilo de vida do paciente, como atividade física, consumo de álcool, tabaco, maconha, cocaína ou crack, e outros usam drogas. Também foram avaliadas reações adversas a medicamentos e outras comorbidades 
que os pacientes a presentaram durante o estudo.

O MMAS-4 foi aplicado em dois momentos, imediatamente antes do IF e após três meses para reavaliar a adesão à medicação dos pacientes e se O IF influenciou a adesão. A coleta de informações ocorreu individualmente em uma sala reservada. Após aplicação do questionário sociodemográfico e da escala MMAS-4, ocorreu IF. O instrumento é constituído com quatro perguntas dicotômicas (1) Nunca se esqueceu de tomar remédios; (2) Sempre descuidado em tomar remédios; (3) Para de tomar medicamentos quando se sente melhor; (4) Para de tomar medicamentos quando se sente pior. Os resultados são apresentados em uma pontuação que varia de $\mathrm{O}$ a 4, e os autores sugeriram três niveis de adesão à medicação com base nessa pontuação: alta, média e baixa aderência com 0, 1-2 e 3-4 pontos, respectivamente. Uma definição dicotômica de aderência com base no MMAS-4 também é comumente usada com 0 pontos, indicando adesão perfeita e 1+ pontos indicando algum nível de não adesão ${ }^{29}$.

As IF foram realizadas com vídeos educativos e rodas de conversas de fácil compreensão para trocar experiências entre os próprios pacientes e a equipe de saúde. Os tópicos abordados foram: papel do farmacêutico no CAPSad, administração correta de medicamentos, uso racional de medicamentos, riscos relacionados ao paciente com 0 tratamento e a não adesão correta, plantas medicinais e drogas ilícitas, uso concomitante de medicamentos psicotrópicos, riscos de automedicação e armazenamento adequado dos medicamentos.

Figuras, tabelas e pictogramas também foram utilizados para ilustrar a administração de medicamentos, a fim de facilitar o entendimento da dosagem e do regime terapêutico pelos pacientes. A tabela é composta por imagens e símbolos que indicam manhã, almoço, tarde e noite (pictogramas), com a dose do medicamento a ser tomado em cada período e como deve ser administrado. Além de promover atividades educacionais que contribuem para aumentar a adesão aos medicamentos, outras ações foram realizadas para melhorar a saúde dos pacientes, como: informar, orientar e educá-los, bem como seus familiares ou cuidadores sobre comportamentos saudáveis e uso racional de medicamentos.

\section{Análise estatística}

A análise dos dados foi realizada no Statistical Package for the Social Sciences SPSS 20.0. Para avaliar a significância estatística $(p \leq 0,05)$ da associação entre as variáveis dicotômicas, foi utilizado o teste de McNemar e, para as ordinais, o teste de Wilcoxon Signed Rank. Um intervalo de confiança de 95\% foi considerado para todos os testes estatísticos e as diferenças foram consideradas estatisticamente significativas se o valor de p foi menor que 0,05.

\section{Resultados}

Todos os pacientes do CAPSad que concordaram em participar do estudo assinaram o Termo de Consentimento Livre e Esclarecido. No entanto, apenas 30 pacientes ativos completaram todas as etapas do estudo, e esse número foi utilizado para a análise estatística dos dados. A exclusão de pacientes do estudo está relacionada à ausência ou estar sob a influência de álcool ou outras drogas nas semanas de estudo, ou mesmo ter 
desistido de participar sem justificativa. Alguns pacientes acreditam que falharam em melhorar a adesão aos medicamentos e, portanto, desistiram de participar da segunda etapa do estudo, a reaplicação da escala MMAS-4. Além disso, deve-se notar que o CAPSad é um serviço de portas abertas, sem hospitalização ou participação obrigatória. Os pacientes têm autonomia para entrar e sair do tratamento a qualquer momento. Outro ponto a ser considerado é que o número médio de pacientes ativos, que frequentam regularmente todas as atividades semanais, é de cerca de 50. Considerando nosso $n=30$ pacientes, é possível concluir que as perdas não impactam na avaliação global dos achados.
A idade dos pacientes variou de 24 a 63 anos, $80 \%$ dos pacientes tinham entre 30 e 60 anos e 93,3\% da amostra era do sexo masculino, como ocorre em outros CAPSad, e de acordo com dados de outros estudos 31,32. O número de analfabetos ou com o ensino médio incompleto foi de $90 \%$ e o desempregado ou número de aposentados foi de 53,3\% (TABELA 1). Quanto ao comportamento em saúde, 66,7\% dos pacientes nunca ou raramente praticavam atividades físicas (TABELA 2). Consumo de álcool (76,7\%) e tabagismo $(66,6 \%)$ foram as substâncias mais utilizadas pelos pacientes, seguidos de maconha (36,7\%), cocaína (13,3\%) e crack (3\%) (TABELA 3).

TABELA 1 - Caracterização dos dependentes químicos CAPSad segundo o Perfil Sociodemográfico $(n=30)$

\begin{tabular}{llll}
\hline Características & Variáveis & N & $\%$ \\
\hline Gênero & Feminino & 2 & 6,7 \\
& Masculino & 28 & 93,3 \\
& $18-29$ & 4 & 13,3 \\
& $30-39$ & 8 & 26,7 \\
Faixa Etária & $40-49$ & 8 & 26,7 \\
& $50-59$ & 8 & 26,7 \\
& 60 ou mais & 1 & 3,3 \\
& Ausente & 1 & 3,3 \\
& Analfabeto & 17 & 56,7 \\
& Ensino & & \\
Escolaridade & Fundamental & 10 & 33,3 \\
& Incompleto & & \\
& Ensino & & 6,7 \\
& Fundamental & 2 & \\
& Completo & &
\end{tabular}




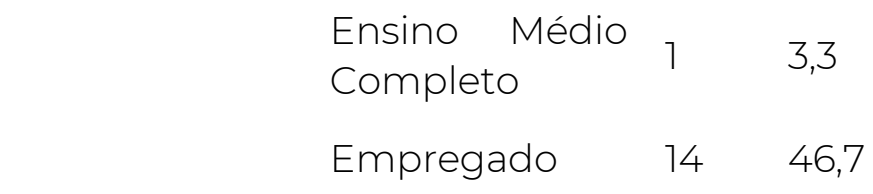

$\begin{array}{lll}\text { Situação } & \text { Desempregado } 12 \quad 40,0\end{array}$

Aposentado $\quad 4 \quad 13,3$

Fonte: Centro de Atenção Psicossocial para álcool e outras Drogas, Lagarto-SE.

TABELA 2 - Hábitos relacionados à prática de atividades físicas dos usuários do CAPSad $(n=30)$

\begin{tabular}{llll}
\hline Características & Variáveis & N & $\%$ \\
\hline & Sim, raramente & 3 & 10,0 \\
& Sim, 1 vez / semana & 5 & 16,7 \\
Prática & atividade & Sim, 3 a 4 vezes / 3 & 10,0 \\
física & Semana & & \\
& Sim, >5 vezes / semana & 2 & 6,7 \\
& Não & 17 & 56,7
\end{tabular}

Fonte: Centro de Atenção Psicossocial para álcool e outras Drogas, Lagarto-SE.

TABELA 3 - Consumo de drogas lícitas e ilícitas pelos usuários do CAPSad ( $n=30$ )

\begin{tabular}{llll}
\hline Características & Variáveis & N & $\%$ \\
\hline & Sim, <5 cigarros / dia & 4 & 13,3 \\
& Sim, de 5 a 10 cigarros / dia & 7 & 23,3 \\
Consumo de Tabaco & Sim, de 11 a 20 cigarros / dia & 7 & 23,3 \\
& Sim, >20 cigarros / dia & 2 & 6,7 \\
& Não & 10 & 33,3 \\
& Total & 30 & 100,0 \\
& Sim, 1 vez / semana & 7 & 23,3 \\
& Sim, 3 vezes / semana & 3 & 10,0 \\
Sim, 1 vez / dia & 2 & 6,7
\end{tabular}




\begin{tabular}{|c|c|c|c|}
\hline & Sim, >1 vez / dia & 11 & 36,7 \\
\hline & Não & 7 & 23,3 \\
\hline & Total & 30 & 100,0 \\
\hline & Sim, Raramente & 1 & 3,3 \\
\hline $\begin{array}{l}\text { Consumo } \\
\text { Cannabis }\end{array}$ & Sim, 1 cigarro / semana & 2 & 6,7 \\
\hline & Sim, >1 cigarro / dia & 8 & 26,7 \\
\hline & Não & 19 & 63,3 \\
\hline & Sim, Raramente & 1 & 3,3 \\
\hline $\begin{array}{l}\text { Consumo } \\
\text { Cocaína }\end{array}$ & Sim, 1 vez / semana & 2 & 6,7 \\
\hline & Sim, 3 vezes / semana & 1 & 3,3 \\
\hline & Não & 26 & 86,7 \\
\hline Consumo de Crack & Sim, Raramente & 4 & 3 \\
\hline & Não & 26 & 86,7 \\
\hline
\end{tabular}

Fonte: Centro de Atenção Psicossocial para álcool e outras Drogas, Lagarto-SE.

Com a primeira aplicação do Instrumento MMAS-4, antes do IF, foi determinado que apenas $10 \%$ dos pacientes incluídos no estudo apresentavam alta adesão, 13,3\% apresentavam adesão média e 76,6\% apresentavam baixa adesão. Três meses, após o desenvolvimento das atividades do IF, o MMAS-4 foi reaplicado com os mesmos pacientes; e uma melhoria significativa foi encontrada na adesão à medicação. O número de pacientes que apresentaram alta adesão à medicação mudou de 10\% para 23,3\% ( $p<0,01)$ após o IF. A proporção de pacientes com adesão média melhorou de 13,3\% para 26,6\% ( $\mathrm{p}$ $<0,01)$, e a proporção de pacientes com baixa adesão reduziu de $76,7 \%$ para $50 \%$ ( $p<0,05)$. Analisando as questões do MMA-4 individualmente, houve diferenças significativas em todas as questões ( $p=0,008 ; 0,039 ; 0,021 ; 0,039$ ), respectivamente (TABELA 4). 
TABELA 4 - Caracterização dos usuários do CAPSad segundo a adesão ao tratamento medicamentoso através da MMM-4 $(n=30)$

\section{Antes IF Depois IF}

\begin{tabular}{cccccc}
\hline Perguntas & Sim (\%) & Não (\%) & Sim (\%) & Não (\%) & $p^{*}$ \\
\hline $\begin{array}{c}\text { 1) Você já se esqueceu de tomar } \\
\text { seus medicamentos psicotrópicos? }\end{array}$ & $19(63,3)$ & $11(36,7)$ & $11(36,7)$ & $19(63,3)$ & 0,008 \\
\hline $\begin{array}{c}\text { 2) Você já foi descuidado ao tomar } \\
\text { seus medicamentos psicotrópicos? }\end{array}$ & $17(56,7)$ & $13(43,3)$ & $10(33,3)$ & $20(66,7)$ & 0,039 \\
\hline $\begin{array}{c}\text { 3) Você para de tomar seus } \\
\text { medicamentos quando se sente } \\
\text { melhor? }\end{array}$ & $20(66,7)$ & $10(33,3)$ & $12(40,0)$ & $18(60,0)$ & 0,027 \\
$\begin{array}{c}\text { 4) Você para de tomar seus } \\
\text { medicamentos quando se sente } \\
\text { pior? }\end{array}$ & $21(70,0)$ & $9(30,0)$ & $13(43,3)$ & $17(56,7)$ & 0,039 \\
& & & & & \\
\end{tabular}

*McNemar Test. Fonte: Centro de Atenção Psicossocial para álcool e outras Drogas, Lagarto-SE.

Os medicamentos utilizados pelos pacientes incluídos neste estudo estão descritos na Tabela 5. A monoterapia psicotrópica foi prescrita para 36,7\% dos pacientes, $36,7 \%$ deles usavam dois psicotrópicos e 23,3\% usavam três ou mais psicotrópicos durante o estudo. Os medicamentos psicotrópicos mais prescritos foram Diazepam (24,6\%), seguidos por Carbamazepina e Haloperidol (17,5\%), Prometazina e Levomepromazina (14\%) (TABELA 5).

TABELA 5: Medicamentos Psicotrópicos Prescritos por Paciente $(n=30)$

\begin{tabular}{cccc}
\hline & Variável & $\mathrm{N}^{\circ}$ & $\mathrm{N} \%$ \\
\hline $\begin{array}{c}\text { Quantidade de } \\
\text { psicotrópicos } \\
\text { prescritos }\end{array}$ & 1 & 11 & 36,7 \\
& 2 & 11 & 36,7 \\
& Não Respondeu & 4 & 13,3 \\
& Total & 3 & 10,0 \\
& Carbamazepina & 30 & 100,0 \\
\hline & Clonazepam & 2 & $3,5 \%$
\end{tabular}




\begin{tabular}{|c|c|c|c|}
\hline & Cloridrato de Amitriptilina & 1 & $1,8 \%$ \\
\hline & Decanoato de Haloperidol & 1 & $1,8 \%$ \\
\hline Frequência no uso & Diazepam & 14 & $24,6 \%$ \\
\hline \multirow[t]{6}{*}{ de psicotrópicos } & Fenobarbital & 2 & $3,5 \%$ \\
\hline & Haloperidol & 10 & $17,5 \%$ \\
\hline & Levomepromazina & 8 & $14,0 \%$ \\
\hline & Prometazina & 8 & $14,0 \%$ \\
\hline & Cloridrato de Fluoxetina & 1 & $1,8 \%$ \\
\hline & Total & 57 & $100,0 \%$ \\
\hline
\end{tabular}

Fonte: Centro de Atenção Psicossocial para álcool e outras Drogas, Lagarto-SE.

\section{Discussão}

Os resultados deste estudo mostram que, após a IF, o número de pacientes que melhoraram a adesão à medicação aumentou significativamente em relação ao apresentado pelos mesmos pacientes antes da IF. O paciente deve aderir ao medicamento prescrito e seguir as orientações da equipe de saúde para sua recuperação. A não adesão ou baixa adesão de pacientes no CAPSad que usavam medicação psicotrópica antes da IF atingiu quase 90\%. A maioria dos pacientes com baixa adesão apresentou comportamento não intencional, ou seja, esquece de tomar os medicamentos, faz uso inadequado ou confunde o tempo ou a dose do uso dos medicamentos.

Segundo Jin e colaboradores (2008), aqueles pacientes que se comportam intencionalmente como tomar a decisão de abandonar o tratamento prescrito, geralmente o fazem devido à falta de conhecimento ou informações adequadas sobre o medicamento ${ }^{33}$. Miasso e col. (2009) relatam que, em uma entrevista de pacientes com transtorno mental, os pacientes afirmaram que a não adesão à medicação ocorre de forma não intencional, mesmo confirmando que entenderam as recomendações da equipe de saúde ${ }^{34}$. A falta de adesão à medicação é um impedimento para as metas de realização terapêutica e pode ser uma fonte de frustração para pacientes e profissionais de saúde ${ }^{35}$.

A baixa adesão prejudica a evolução clínica do paciente e sua qualidade de vida, causando resultados adversos, como aumento da morbimortalidade ${ }^{36}$. Nos pacientes com transtorno afetivo bipolar, quando o tratamento não é realizado corretamente, leva ao aumento de episódios de mania e transtornos depressivos, hospitalizações e até suicídio, prejudicando a melhoria e a qualidade de vida de ambos, pacientes e seus familiares, além do aumento de custos para o sistema de saúde ${ }^{37}$. Um estudo realizado na Arábia Saudita mostra que a adesão a medicamentos antidepressivos é uma etapa essencial no tratamento do paciente com distúrbios psicológicos, e vários fatores associados às crenças e satisfações do paciente podem contribuir para o não cumprimento do tratamento com medicamentos psicotrópicos ${ }^{38}$. 
As tabelas foram preparadas com a medicação correta; dosagem, tempo e como tomar o medicamento, foi enfatizada a importância da participação do paciente e da família nas oficinas terapêuticas. Ferreira e col. (2015) disseram que a família é de suma importância para a reabilitação do paciente e a adesão à medicação, participando ativamente de reuniões familiares ou incentivando o usuário a participar. No entanto, quando não existe esse apoio, são observadas dificuldades nos pacientes em manter o tratamento medicamentoso. $O$ autor cita, ainda, que a família precisa entender que a dependência química é uma doença crônica, requer tratamento e não é falta de moralidade ou irresponsabilidade do dependente químico ${ }^{39}$. Segundo o estudo de Boston, a adesão à medicação era difícil quando a pessoa afetada não tinha cônjuge ou companheiro, sofria de reações adversas ou era mais jovem ${ }^{40}$.

Observou-se na população estudada que, quando houve participação familiar, a adesão aos medicamentos do paciente melhorou significativamente. Embora essa variável não tenha sido medida estatisticamente em nosso estudo, foi possivel observar que os pacientes que apresentaram melhora significativa nos escores do MMAS-4 foram os que os parentes mais participaram das reuniões familiares do CAPSad, realizadas uma vez por mês. A participação da família na recuperação do paciente é um dos pilares de tratamento propostos nesta instituição, e a rede de apoio à família é uma prioridade nesse processo. A prescrição de psicofármacos a usuários de drogas em tratamento no CAPSad é sintomática e, segundo Bezerra e colaboradores, seu uso precisa estar limitado ao extremamente necessário, devendo sempre levar em consideração a relação risco-benefício potencial do fármaco justifica seu emprego ${ }^{47}$.

A Escala de Adesão à Medicação Morisky com quatro itens (MMAS-4) é um dos métodos de adesão à medicação mais comumente usados, com 0,83 de confiabilidade, criado com o objetivo de determinar a adesão à medicação antihipertensiva ${ }^{29}$. Optou-se por utilizar neste estudo apenas a Escala de Morisky, devido à dificuldade de aplicar instrumentos em pacientes com transtornos relacionados ao uso de álcool e problemas relacionados a substâncias. Nossos resultados mostraram que 0 instrumento MMAS-4 é um instrumento extremamente útil para avaliação e monitoramento do uso de medicamentos pelos pacientes do CAPSad investigados neste estudo e proporcionou um trabalho individualizado de acordo com as respostas do paciente.

Entre os pacientes avaliados, o medicamento psicotrópico mais utilizado foi o diazepam, seguido de carbamazepina e fenobarbital. Esses medicamentos foram prescritos principalmente para suprimir a ansiedade e, consequentemente, o vício ou fissura do paciente, bem como abolir convulsões. Por outro lado, esses medicamentos causam muitos efeitos adversos, incluindo tontura, ataxia e sonolência, que levam ao baixo grau de adesão ao medicamento. O diazepam é o medicamento mais prescrito, pois faz parte da lista de medicamentos essenciais do governo, disponível gratuitamente, tornando-o mais acessivel.

Uma das limitações do estudo foi o número pequeno de participantes e por ter sido conduzido em apenas uma instituição. O tempo de intervenção Farmacêutica de 3 meses também pode 
ser considerada insuficiente, fato superado pelos excelentes resultados obtidos. Há necessidade de uma assistência farmacêutica continuada para que se mantenha a adesão terapêutica em níveis aceitáveis e que outros cuidados possam ser implantados.

\section{Conclusão}

O desempenho da intervenção farmacêutica foi suficiente para aumentar significativamente a adesão à medicação do paciente. A realização de atividades educacionais, distribuição de material didático para apoiar a manutenção correta dos tratamentos prescritos, somada à participação da família, demonstrou ser instrumentos muito eficazes na mudança de comportamento do dependente químico em relação à adesão aos medicamentos e redução de danos. Também é necessário que os médicos simplifiquem os esquemas de dosagem, adaptando-os ao estilo de vida do paciente e, sempre que possivel, os farmacêuticos devem procurar resolver os problemas reais relacionados à medicação e prevenir possíveis efeitos colaterais ${ }^{42}$.

O impacto positivo do atendimento colaborativo multidisciplinar em pacientes do CAPSad, demonstraram bons resultados, foram contínuos e persistentes ao longo do estudo. A abordagem de atendimento colaborativo multidisciplinar pareceu ser eficaz no gerenciamento de pacientes de alto risco com dependência química descontrolada, e não apenas nos cuidados usuais.
A escassez de atividades promovidas pelos farmacêuticos nos CAPS direcionadas aos usuários ou mesmo a

falta de registro de intervenções atestam a deficiência do trabalho assistencial, colocando em questionamento o impacto que a falta dessa atividade pode promover na promoção e recuperação da saúde ${ }^{43}$.

\section{Declaração de conflito de interesses}

Os autores declaram não terem nenhum conflito de interesses na condução e publicação deste artigo.

\section{Agradecimentos}

- Secretaria Municipal de Saúde de Lagarto, especialmente à equipe do Centro de Assistência Psicossocial de álcool e outras drogas e, principalmente, a todos os usuários que participaram das atividades propostas.

\section{Referências}

1. WHO, Global status report on alcohol and health. World Health Organization, 2014. Disponivel em: <https://www.who.int/substance_a buse/publications/global_alcoholr eport /en/

2. RONZANI, T. M., FURTADO, E. F. Estigma social sobre o uso de álcool. Jornal Brasileiro de Psiquiatria, v. 59, n. 4, p. 326-332, 2010.

3. IARC: International Agency for Research on Cancer. World Health Organization, 2020. Disponível em:<https://www.iarc.fr/

4. UK, Chief Medical Officers. Alcohol Guidelines Review: Summary of the proposed new guidelines. 
World Health Organization, 2015.

Disponivel em:

<https://assets.publishing.

service.gov.uk/government/upload s/system/uploads/attachment_dat a/file/489795/summary .pdf

5. WOOD, A. M., \& Emerging Risk Factors Collaboration/EPICCVD/UK Biobank Alcohol Study Group. Risk thresholds for alcohol consumption: combined analysis of individual-participant data for 599912 current drinkers in 83 prospective studies. The Lancet, $v$. 391, p.1513-23, 2018.

6. UNODOC, United Nations Office on Drugs and Crime. World Drug Report 2016. United Nations Publication, n. E.16.XI.7, 2016. Disponível em: <https://www.unodc.org/wdr 2016/

7. GOLDSTEIN, R. Z., VOLKOW, N. D. Drug addiction and its underlying neurobiological basis:

neuroimaging evidence for the involvement of the frontal cortex. American Journal of Psychiatry, $v$. 159, n. 10, p. 1642-1652, 2002.

8. HATSUKAMI, D. K., FISCHMAN, M. W. Crack cocaine and cocaine hydrochloride. Are the differences myth or reality? Journal of the American Medical Association, v. 276, n. 19, p. 1580-1588, 1996.

9. UNODOC, United Nations Office on Drugs and Crime. World Drug Report 2017. United Nations Publication, n. E.17.XI.6, 2017. Disponível em: <http://www.unodc.org/wdr 2017/

10. Johnson J, Wagner J. Trump declares the opioid crisis a public health emergency. The Washington Post, out., 2017.
Disponivel

em:<https://www.washingtonpost. com/news/ postpolitics/wp/2017/10/26/trumpplans-to-declare-the-opioid-crisisa-public-health-em ergency/

11. BASTOS, F. I. P. M., VASCONCELLOS, M.T. L., DE BONI, R. B., REIS, N. B., COUTINHO, C. F. S. ARCA: III Levantamento Nacional sobre o uso de drogas pela população brasileira. Fiocruz, 2017, 528p. Disponível em: <https://www.arca.fiocruz.br/ handle/icict/34614.

12. BASTOS, F. I., BERTONI, N. Pesquisa Nacional do Uso do Crack e outras Drogas. Fiocruz, 2013. Disponível em: < https://app.uff.br/observatorio/uplo ads/Pesquisa_

Nacional_sobre_uso_de_crack_e_o utras_drogas.pdf

13. BRASIL: Ministério da Saúde. Portaria $n^{\circ} 130$, de 26 de janeiro de 2012(*). Redefine o Centro de Atenção Psicossocial, Álcool e outras Drogas 24 horas (CAPS AD III) e os respectivos incentivos financeiros. Disponível em: < http://www.saude.gov.br/images/p df/ 2015/marco/10/PORTARIA-13026--JANEIRO-2012.pdf

14. MORAES, M. O modelo de atenção integral à saúde para tratamento de problemas decorrentes do uso de álcool e outras drogas: percepções de usuários, acompanhantes e profissionais. Ciência \& Saúde Coletiva, v.13, n.1, p. 121-133, 2008.

15. KNUDSEN, H. K., DUCHARME, L. J., ROMAN, P. M. The use of antidepressant medications in substance abuse treatment: The 
public-private distinction, organizational compatibility, and the environment. Journal of Health and Social Behavior, v. 48, p. 195-210, 2007.

16. FOULDS, J.A., ROUCH, S., SPENCE, J., MULDER, R.T., SELLMAN, J.D. Prescribed Psychotropic Medication Use in Patients Receiving Residential Addiction Treatment. Alcohol and Alcoholism. v. 51, n. 5, p. 622-623, 2016.

17. KOOB, G. F., VOLKOW, N. D. Neurocircuitry of Addiction. Neuropsychopharmacology, v. 35, p. 217-238, 2010.

18. BUTELMAN, E., KREEK, M. J. Medications for substance use disorders (SUD): Emerging approaches. Expert Opinion of Emerging Drugs, v. 22, n. 4, p. 301315, 2017.

19. SOUZA, J., KANTORSKI, L. P. A rede social de indivíduos sob tratamento em um CAPS ad: o ecomapa como recurso. Revista da Escola de Enfermagem da USP, v.43, n.2, p.373-383, 2009.

20. SCHMIDT, M. I., DUNCAN, B. B., SILVA, G. A., MENEZES, A.M., MONTEIRO, C.A., BARRETO, S.M., CHOR, D., MENEZES, P.R. Chronic non-communicable diseases in Brazil: burden and current challenges. The Lancet, v. 377, n. 9781, p. 1949-1961, 2011.

21. DAVIS, D. R., KURTI, A. N., SKELLY, J. M., REDNER, R., WHITE, T. J., HIGGINS, S. T. A review of the literature on contingency management in the treatment of substance use disorders, 2009-
2014. Preventive Medicine, v. 92, p. 36-46, 2016.

22. ABOUJAOUDE, E., SALAME, W. O. Naltrexone: a pan-addiction treatment? CNS Drugs, v. 30, n. 8, p. 719-733, 2016.

23. FALCK, R. S., WANG, J., CARLSON, R. G. Among long-term crack smokers, who avoids and who succumbs to cocaine addiction? Drug Alcohol Dependence, v. 98, n. 1-2, p. 24-29, 2008.

24. DUNN, J., LARANJEIRA, R. Cocaine-profiles, drug histories, and patterns of use of patients from Brazil. Substance Use and Misuse, v. 34, n. 11, p. 1527-1548, 1999

25. GOSSOP, M., MARSDEN, J., STEWART, D., KIDD, T. Changes in use of crack cocaine after drug misuse treatment: 4-5 year followup results from the National Treatment Outcome Research Study (NTORS). Drug Alcohol Dependence, v. 66, n. 1, p. 21-28, 2002.

26. CUMMING, C., TROEUNG, L., YOUNG, J. T., KELTY, E., PREEN, D. $B$. Barriers to accessing methamphetamine treatment: a systematic review and metaanalysis. Drug Alcohol Dependence, v. 168, p. 263-273, 2016.

27. PADWA, H., URADA, D., GAUTHIER, P., RIECKMANN, T., HURLEY, B., MACPHAIL, D. C., RAWSON, R. A. Organizing publicly funded substance use disorder treatment in the United States: moving toward a service system approach. Journal of Substance Abuse Treatment, v. 69, p. 9-18, 2016. 
28. PINSKY, I., BERNAL, C., VUOLO, L., NEIGHBORS, C. Introducing care management to Brazil's alcohol and substance use disorder population. Revista Brasileira de Psiquiatria, v.40, n.3, 2018.

29. MORISKY, D. E., GREEN, L. W., LEVINE, D. M. Concurrent and predictive validity of a selfreported measure of medication adherence. Medical Care. v. 24, n. 1, p. 67-74, 1986.

30. REMONDI, F. A., CABRERA, M. A. S., SOUZA, R. K. T. Não adesão ao tratamento medicamentoso contínuo: prevalência e determinantes em adultos de 40 anos e mais. Cadernos de Saúde Pública, v.30, n. 1, p. 126-36, 2014.

31. DI PIETRO, G., SILVA, R. M. R., SANTOS, J. C., SANTANA, T. S., REZENDE, B. A. M., SANTANA, A. P. A., OLIVEIRA, M. A., SANTOS, C. B., SANTOS, J. S., SOUZA, A. A., SANTOS, J. C. S., SILVA, R. C. R., SANTOS, L. K. L., SOUZA, N. J. P., LIMA, F. R. S., SOUZA, I. C. F., CEZAR, C. F., ALCANTARA, J. C., BARROS, R. G., SILVA, D. S., FREIRE, J. M., SANTOS, S. C., LIMA, C. M. Features and factors associated with drinking problems or illegal drugs use: a cross sectional cohort study with patients of a psychosocial care service. Applied Clinical Research, Clinical Trials and Regulatory Affairs, v. 3, n. 3, p. 192-200, 2016.

32. MONTEIRO, C. F. S., FÉ, L. C. M., MOREIRA, M. A. C., ALBUQUERQUE, I. E. M., SILVA, M. G., PASSAMANI, M. C. Perfil sociodemográfico e adesão ao tratamento de dependentes de álcool em CAPS-ad do Piauí.
Escola Anna Nery, v. 15, n. 1, p. 9095, 2011.

33. JIN, J., SKLAR, G. E., OH, V. M. S., LI, S. C. Factors affecting therapeutic compliance: A review from the patient's perspective. Therapeutics and Clinical Risk Management, v. 4, n. 1, p. 269-286, 2008.

34. MIASSO, A. I., MONTESCHI, M., GIACCHERO, K. G.. Bipolar affective disorder: medication adherence and satisfaction with treatment and guidance by the health team in a mental health service. Revista Latino-Americana de Enfermagem, v. 17, n. 4, p. 1-9, 2009.

35. JESUS, E. S., AUGUSTO, M. A. O., GUSM, J., MION JÚNIOR, D., ORTEGA, K., PIERIN, A. M. G. Perfil de um grupo de hipertensos: aspectos biossociais, conhecimentos e ades, 0 ao tratamento. Acta Paulista de Enfermagem, v. 21, n. 1, p. 59-65, 2008.

36. PITTMAN, D. G., TAO, Z., CHEN, W., STETTIN, G. D. Antihypertensive medication adherence and subsequent healthcare utilization and costs. American Journal of Management Care, v. 16, n. 8, p. 568-76, 2010.

37. GREENHOUSE, W. J., MEYER, B., JOHNSON, S.L.. Coping and medication adherence in bipolar disorder. Journal of Affect

Disorder, v. 59, n. 3, p. 237-241, 2000.

38. ALJUMAH, K., HASSALI, A.A., ALQHATANI, S. Examining the relationship between adherence and satisfaction with antidepressant treatment. 
Neuropsychiatr Disease

Treatment, v. 10, p. 1433-1438, 2014.

39. FERREIRA, A. C. Z., BORBA, L. O., CAPISTRANO, F. C., CZARNOBAY, J., MAFTUM, M. A. Fatores que interferem na adesão ao tratamento de dependência química: percepção de profissionais de saúde. Revista Mineira de Enfermagem, v. 19, n. 2, p. 150-156, 2015.

4O. BERRY, D. L., BLONQUIST, T. M., HONG, F., HALPENNY, B., PARTRIDGE, A. H. Self-reported adherence to oral cancer therapy: relationships with symptom distress, depression, and personal characteristics. Patient Preference and Adherence, v. 9, p. 1587-1592, 2015.
41. BEZERRA, I. C., MORAIS, J. B., PAULA, M. L., SILVA, T. M. R., JORGE, M. S. B. Uso de psicofármacos na atenção psicossocial: uma análise à luz da gestão do cuidado. Saúde em Debate. v. 40, n. 110, p. 148-161, 2016.

42. BRAGA, D. S., BORGES, K. D. M., IODES, A. M. F., FREITAS R. M.

Estudo do uso racional medicamentos por usuários do centro de atenção psicossocial CAPS VI. Infarma, v. 17, n. 7-9, p. 7477. 2005.

43. SILVA, S. N., LIMA, M. G. Assistência Farmacêutica na Saúde Mental: um diagnóstico dos Centros de Atenção Psicossocial. Ciência \& Saúde Coletiva, v. 22, n. 6, p. 20252036, 2017. 